Authors

David J. Jones, Scott A. Diddams, Jinendra K. Ranka, Andrew Stentz, Robert S. Windeler, John L. Hall, and Steven T. Cundiff 


\title{
Carrier-Envelope Phase Control of Femtosecond Mode-Locked Lasers and Direct Optical Frequency Synthesis
}

\author{
David J. Jones, ${ }^{1 *}$ Scott A. Diddams, ${ }^{1 *}$ Jinendra K. Ranka, ${ }^{2}$ \\ Andrew Stentz, ${ }^{2}$ Robert S. Windeler, ${ }^{2}$ \\ John L. Hall, ${ }^{1 *}$ Steven T. Cundiff ${ }^{1 * \dagger}$
}

\begin{abstract}
We stabilized the carrier-envelope phase of the pulses emitted by a femtosecond mode-locked laser by using the powerful tools of frequency-domain laser stabilization. We confirmed control of the pulse-to-pulse carrier-envelope phase using temporal cross correlation. This phase stabilization locks the absolute frequencies emitted by the laser, which we used to perform absolute optical frequency measurements that were directly referenced to a stable microwave clock.
\end{abstract}

Progress in femtosecond pulse generation has made it possible to generate optical pulses that are only a few cycles in duration (1-4). This has resulted in rapidly growing interest in controlling the phase of the underlying carrier wave with respect to the envelope $(1,5-7)$. The absolute carrier phase is normally not important in optics; however, for such ultrashort pulses, it can have physical consequences $(6,8)$. Concurrently, mode-locked lasers, which generate a train of ultrashort pulses, have become an important tool in precision optical frequency measurement (9-14). There is a close connection between these two apparently disparate topics. We exploited this connection to develop a frequency domain technique that stabilizes the carrier phase with respect to the pulse envelope. Using the same technique, we performed absolute optical frequency measurements using a single mode-locked laser with the only input being a stable microwave clock.

Mode-locked lasers generate a repetitive train of ultrashort optical pulses by fixing the relative phases of all of the lasing longitudinal modes (15). Current mode-locking techniques are effective over such a large bandwidth that the resulting pulses can have a duration of $6 \mathrm{fs}$ or shorter, i.e., approximately two optical cycles (2-4). With such ultrashort pulses, the relative phase between peak of the pulse envelope and the underlying electric-field carrier wave becomes relevant. In general, this phase is not constant from pulse to pulse because the group and phase

1 JILA, University of Colorado and National Institute of Standards and Technology, Boulder, CO 80309-0440, USA. ${ }^{2}$ Bell Laboratories, Lucent Technologies, Murray Hill, NJ 07733, USA.

*These authors contributed equally to this work. $\dagger$ To whom correspondence should be addressed. Email: cundiffs@jila.colorado.edu velocities differ inside the laser cavity (Fig. 1A). To date, techniques of phase control of femtosecond pulses have employed time domain methods (5). However, these techniques have not used active feedback, and rapid dephasing occurs because of pulse energy fluctuations and other perturbations inside the cavity. Active control of the relative carrierenvelope phase prepares a stable pulse-topulse phase relation, as presented below, and will dramatically impact extreme nonlinear optics.

Although it may be natural to think about the carrier-envelope phase in the time domain, it is also apparent in a high-resolution measurement of the frequency spectrum. The output spectrum of a mode-locked laser consists of a comb of optical frequencies separated by the repetition rate. However, the comb frequencies are not necessarily integer multiples of the repetition rate; they may also have an offset (Fig. 1B). This offset is due to the difference between the group and phase velocities. Control of the carrier-envelope phase is equivalent to control of the absolute optical frequencies of the comb, and vice versa. This means that the same control of the carrier-envelope phase will also result in a revolutionary technique for optical frequency metrology that directly connects the microwave cesium frequency standard to the optical frequency domain with a single laser (14).

We used a self-referencing technique to control the absolute frequencies of the optical comb generated by a mode-locked laser. Through the relation between time and frequency described below, this method also

\section{A Time domain}

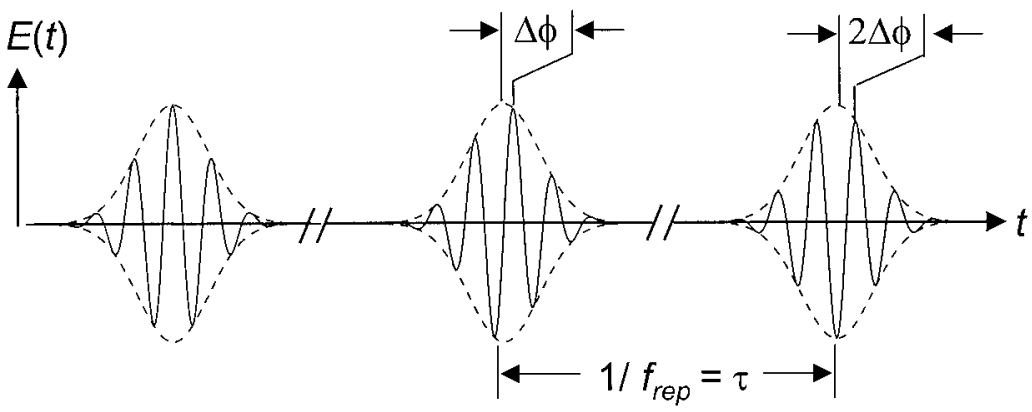

B Frequency domain

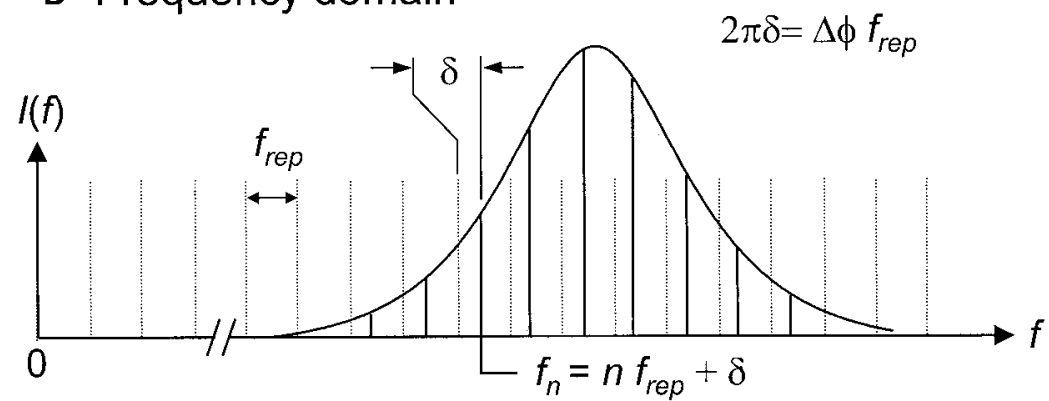

Fig. 1. Time-frequency correspondence and relation between $\Delta \phi$ and $\delta$. (A) In the time domain, the relative phase between the carrier (solid) and the envelope (dotted) evolves from pulse to pulse by the amount $\Delta \phi$. Generally, the absolute phase is given by $\phi=\Delta \phi(t / \tau)+\phi_{0}$, where $\phi_{0}$ is an unknown overall constant phase. (B) In the frequency domain, the elements of the frequency comb of a mode-locked pulse train are spaced by $f_{\text {rep }}$. The entire comb (solid) is offset from integer multiples (dotted) of $f_{\text {rep }}$ by an offset frequency $\delta=\Delta \phi f_{\text {rep }} / 2 \pi$. Without active stabilization, $\delta$ is a dynamic quantity, which is sensitive to perturbation of the laser. Hence, $\Delta \phi$ changes in a nondeterministic manner from pulse to pulse in an unstabilized laser. 


\section{RESEARCH ARTICLES}

serves to stabilize the carrier phase with respect to the envelope. This phase stabilization was verified with temporal cross correlation. The utility of this method for absolute optical frequency metrology was demonstrated by measuring the frequency of a stable singlefrequency laser directly from a microwave clock.

Time versus frequency. The connection between the pulse-to-pulse carrier-envelope phase shift and the absolute frequency spectrum can be understood by considering how the spectrum is built up by a temporal train of pulses $(7,9,14)$. It is easily shown with Fourier transforms that a shift in time corresponds to a phase shift that is linear with frequency; that is, the phase at angular frequency $\omega$ is $\omega t$ for a time shift of $t$. Within a narrow spectral bandwidth, successive pulses interfere, but a signal will be observed only at frequencies where they add constructively, i.e., have a phase shift of $2 n \pi$. For a pulse train with time $\tau$ between pulses, these frequencies are $f_{n}=n / \tau=n f_{\text {rep }}$, where $n$ is an integer and $f_{\text {rep }}=1 / \tau$ is the repetition frequency of the pulse train. Thus, we recover the fact that the frequency spectrum consists of a comb of frequencies spaced by the repetition rate of the pulse train. If we include the pulse-to-pulse phase shift $\Delta \phi$, then the phase difference between successive pulses at angular frequency $\omega$ is now $\omega \tau-\Delta \phi$. Again, for constructive interference, this phase difference is set equal to $2 n \pi$, which shows that now the frequencies are $f_{n}=n f_{\text {rep }}+\delta$, where $2 \pi \delta=\Delta \phi f_{\text {rep }}$. Hence, a pulse-to-pulse phase shift between the carrier and envelope corresponds to an offset of the frequency comb from simple integer multiples of the repetition rate.

In a mode-locked laser, the carrier slips through the envelope as the pulse circulates in the cavity. Because an output pulse is only produced once per cavity round trip, what matters is the accumulated carrier phase, with respect to the envelope, per round trip. If this is an integer multiple of $2 \pi$, then there is no pulse-to-pulse phase shift for the emitted pulses, and the frequencies are all integer multiples of the repetition rate, without an offset. If the accumulated carrier phase is an integer multiple of $2 \pi$ plus a rational fraction of $2 \pi$, then the phase will periodically shift, and the frequency comb will be offset by the repetition rate times the rational fraction. For example, if the pulse-to-pulse phase shift is $2 \pi / 8$, then every eighth pulse will have the same phase, and the frequency offset will be $f_{\text {rep }} / 8$. In the most general case, the phase and frequency offsets are arbitrary. Furthermore, in a free-running laser (even one with its repetition rate locked), the phase-group velocity difference drifts with time.

Self-referencing technique. Choosing to control the carrier-envelope phase by locking the frequency domain offset $\delta$ enabled us to use the powerful techniques developed for stabilization of single-frequency lasers. It is possible to lock the position of the frequency comb to a known optical frequency, such as that of a single-frequency laser (12). However, employing this approach to determine and lock $\delta$ is problematic because the optical frequencies are $>10^{6}$ times the repetition rate. In addition, it introduces the complication of a highly stabilized single-frequency laser, not to mention the uncertainty in the single-frequency continuous wave $(\mathrm{CW})$ laser itself.

A more elegant approach is to use a selfreferencing technique, which is based on comparing the frequency of comb lines on the low-frequency side of the optical spectrum to those on the high-frequency side that have approximately twice the frequency. Let the frequency of comb line $n$, which is on the red side of the spectrum, have a frequency $f_{n}=$ $n f_{\text {rep }}+\delta$. The comb line corresponding to $2 n$, which will be the blue side of the spectrum, will have frequency $f_{2 n}=2 n f_{\text {rep }}+\delta$. We obtain $\delta$ by frequency doubling $f_{n}$ and then taking the difference $2 f_{n}-f_{2 n}=\delta$. To implement the technique in a simple way, we need an optical spectrum that spans a factor of 2 in frequency, known as an optical octave. This is obtained by spectrally broadening the laser pulse in air-silica microstructure fiber (16). Full experimental detail is given below.

Optical frequency metrology techniques. Optical frequencies are preferred for the precision measurements that test fundamental physical theories $(17,18)$. In part, this preference is associated with the very narrow fractional linewidths displayed by optical resonances, allowing precise measurements to be made in relatively short times. For similar reasons, optical resonances will probably be used in future atomic clocks. However, any absolute frequency measurement must be derived from the 9.193-GHz cesium hyperfine transition, which defines the second as one of the SI base units. The frequencies involved and the large ratio between the cesium transition frequency and optical frequencies (a factor of $\sim 5 \times 10^{4}$ times the transition frequency) represent serious obstacles.

Before this work, two techniques have been used to make absolute optical frequency measurements. The first technique is a phasecoherent frequency chain of oscillators that spans from the cesium reference transition to optical frequencies (19). The complexity and difficulty of these chains requires substantial investment and is typically undertaken only at national research facilities. Furthermore, because of their complexity, the chains may not run on a daily basis but rather are typically used to calibrate intermediate standards such as the $\mathrm{HeNe} / \mathrm{I}_{2}$ stabilized laser and $\mathrm{CO}_{2}$ lasers stabilized to $\mathrm{OsO}_{4}$ resonances.

A newer measurement technique involves frequency doubling a single-frequency laser and then measuring the difference between the fundamental and second harmonic by subdividing it. The subdivision may be done by optical bisection $(20,21)$, comb generation (22), or, most typically, a combination of the two $(9,10,17,23)$. The goal of the subdivision is finally to obtain a frequency interval small enough that it can be directly compared to the cesium frequency. A recent series of landmark experiments by Hänsch and co-workers demonstrated that modelocked lasers are the preferred implementation of optical comb generators $(9-11)$. We recently performed absolute frequency measurements using only the comb generated by a mode-locked laser (13). The laser and microstructure fiber were similar to those used here, although the technique is quite different in that the laser comb was not stabilized in position but rather only used to divide down an optical frequency interval.

The self-referencing technique described here is a dramatic step beyond these previous techniques because it uses only a single mode-locked laser and does not need any stabilized single-frequency lasers. We think that it will make precision absolute optical frequency metrology into an easily accessible laboratory tool.

Laser and stabilization. The heart of the experiment is a titanium-doped sapphire (Ti:S) laser (shown in Fig. 2) that is pumped with a single-frequency, frequency-doubled $\mathrm{Nd}: \mathrm{YVO}_{4}$ laser operating at $532 \mathrm{~nm}$. The Ti:S laser generates a $90-\mathrm{MHz}$ pulse train with pulse widths as short as 10 fs using Kerr lens modelocking (2). The output pulse spectrum is typically centered at $830 \mathrm{~nm}$ with a width of $70 \mathrm{~nm}$. For the generation of a 10-fs pulse, the normal dispersion of the Ti:S crystal is compensated by incorporating a pair of fused silica prisms inside the cavity (24). After the second prism, the optical frequencies of the pulse are spatially resolved across the high-reflector mirror; this property will be used to stabilize the absolute frequency of the laser.

We previously noted that the relative carrier-envelope phase $\Delta \phi$ in successive pulses generated by mode-locked lasers is not constant because of a difference between the group and phase velocities inside the cavity. As shown in Fig. 1, this is represented by the frequency offset $\delta$ of the frequency comb from $f_{n=0}=0$. With the pulse repetition rate $f_{\text {rep }}$, the relative phase is related to the offset frequency by $2 \pi \delta=\Delta \phi f_{\text {rep }}$. Thus, by stabilizing both $f_{\text {rep }}$ and $\delta, \Delta \phi$ can be controlled. Toward this end, as shown in Fig. 2 , the high-reflector mirror (behind the prism) is mounted on a piezoelectric transducer tube that allows both tilt and translation. By comparing a high harmonic of the pulse repetition rate with the output of a high-stability radio frequency (RF) synthe- 


\section{RESEARCH ARTICLES}

sizer, a feedback loop can lock the repetition rate $f_{\text {rep }}$ by translating the mirror. Because the pulse spectrum is spatially dispersed across the mirror, tilting of this mirror provides a linear phase change with frequency (i.e., a group delay for the pulse), thereby controlling both the repetition rate and the offset frequency (11). The maximum required tilt angle is $10^{-4} \mathrm{rad}$, substantially less than the beam divergence, so cavity misalignment is negligible.

To stabilize the offset frequency of a single mode-locked laser, without external information, it is useful to generate a full optical octave. The typical spectral output generated by the Ti:S laser used in these experiments spans $70 \mathrm{~nm}$ or $30 \mathrm{THz}$, whereas the center frequency is $\sim 350 \mathrm{THz}$; that is, the spectrum spans much less than a full octave. Propagation through optical fiber is commonly used to broaden the spectrum of modelocked lasers through the nonlinear process of self-phase modulation, based on the intrinsic intensity dependence of the refractive index (the Kerr effect). Optical fiber offers a small mode size and a relatively long interaction length, both of which enhance the width of the generated spectrum. However, chromatic dispersion in the optical fiber rapidly stretches the pulse duration, thereby lowering the peak power and limiting the amount of generated spectra. Although zero dispersion optical fiber at 1300 and $1550 \mathrm{~nm}$ has existed for years, optical fiber that supports a stable, fundamental spatial mode and has zero dispersion near $800 \mathrm{~nm}$ has been available only in the past year. In this work, we employed a recently developed air-silica microstructure fiber that has zero group velocity dispersion at $780 \mathrm{~nm} \mathrm{(16).} \mathrm{The} \mathrm{sustained} \mathrm{high} \mathrm{intensity}$ (hundreds of $\mathrm{GW} / \mathrm{cm}^{2}$ ) in the fiber generates a stable, single-mode, phase-coherent continuum that stretches from 510 to $1125 \mathrm{~nm}$ (at $-20 \mathrm{~dB}$ ) as shown in Fig. 3. Through fourwave mixing processes, the original spectral comb in the mode-locked pulse is transferred to the generated continuum. As described above, the offset frequency $\delta$ is obtained by taking the difference between $2 f_{n}$ and $f_{2 n}$. Figure 2 details this process of frequency doubling $f_{n}$ in a nonlinear crystal and combining the doubled signal with $f_{2 n}$ on a photodetector. The resulting RF heterodyne beat is equal to $\delta$. In actuality, the beat arises from a large family of comb lines, which greatly enhances the signal-to-noise ratio. After suitable processing (described below), this beat is used to actively tilt the high-reflector mirror, allowing us to stabilize $\delta$ to a rational fraction of the pulse repetition rate.

The experimental implementation of the $f-2 f$ heterodyne system is shown in Fig. 2 . The continuum output by the microstructure fiber is spectrally separated into two arms by a dichroic beamsplitter. The visible portion of the continuum (500 to $900 \mathrm{~nm}$, containing $f_{2 n}$ ) is directed through one arm that contains an acousto-optic modulator (AOM). The near-infrared portion of the continuum (900 to $1100 \mathrm{~nm}$, containing $f_{n}$ ) traverses the other arm of the apparatus, passing through a 4-mm-thick $\beta$-barium-borate frequency-doubling crystal. The crystal is angle-tuned to efficiently double at $1040 \mathrm{~nm}$. The beams from the two arms are then mode-matched and recombined. The combined beam is filtered with a 10-nm bandwidth interference filter centered at $520 \mathrm{~nm}$ and focused onto an avalanche photo diode (APD). Approximately $5 \mu \mathrm{W}$ are incident on the APD from the arm containing the AOM, whereas the frequency-doubling arm provides $\sim 1 \mu \mathrm{W}$. The resulting RF beats are equal to $\pm\left(\delta-f_{\mathrm{AOM}}\right)$, where $f_{\mathrm{AOM}}$ is the drive frequency of the $\mathrm{AOM}$ and is generated to be $7 / 8 f_{\text {rep }}$. The RF beats are then fed into a tracking oscillator that phase-locks a voltage-controlled oscilla- tor to the beat to enhance the signal-to-noise ratio by substantially reducing the noise bandwidth. From the tracking oscillator output, we generate an error signal that is programmable to be $(m / 16) f_{\text {rep }}$, thus allowing us to lock the relative carrier-envelope phase from 0 to $2 \pi$ in 16 steps of $\pi / 8$.

Temporal cross correlation. Verification of control of $\Delta \phi$ in the time domain is obtained by interferometric cross correlation between two different, not necessarily adjacent, pulses in the pulse train (5). In fact, we performed a time-averaged cross correlation between pulses $i$ and $i+2$ using the correlator shown in Fig. 4. A multipass cell in one arm of the correlator is used to generate the required 20-ns delay. For the purpose of minimizing dispersion, the beam splitter is a $2-\mu m$-thick polymer pellicle with a thin gold coating. To obtain a well-formed interferogram, we chose the mirror curvatures and their separations to mode-match the output

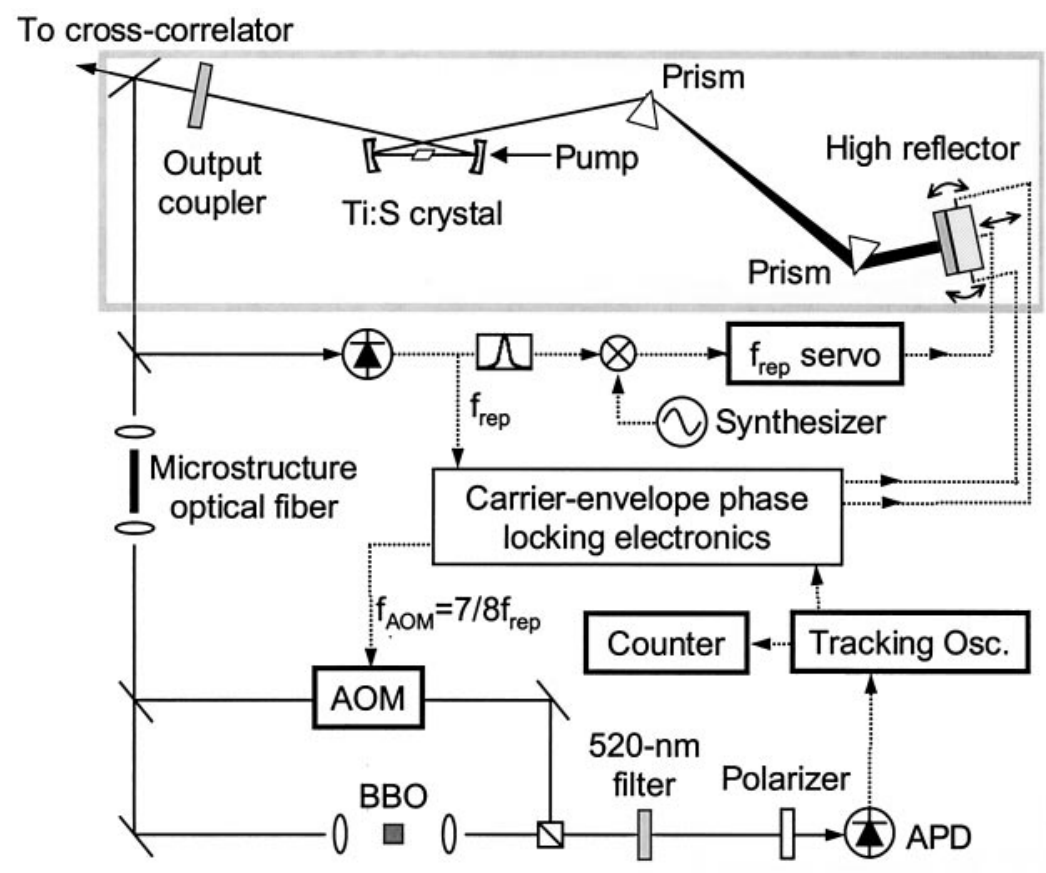

Fig. 2. Experimental setup for locking the carrier-envelope relative phase. The femtosecond laser is located inside the shaded box. Solid lines represent optical paths, and dashed lines show electrical paths. The high-reflector mirror is mounted on a transducer to provide both tilt and translation.

Fig. 3. Continuum generated by air-silica microstructure fiber. Self-phase modulation in the microstructured fiber broadens the output of the laser so that it spans more than one octave. Approximately $25 \mathrm{~mW}$ are coupled into the fiber to generate the displayed continuum. The wavelengths/frequencies denoted by $f_{n}$ and $f_{2 n}$ are used to lock the offset frequency as described in the text. The spectra are offset from each other for clarity.

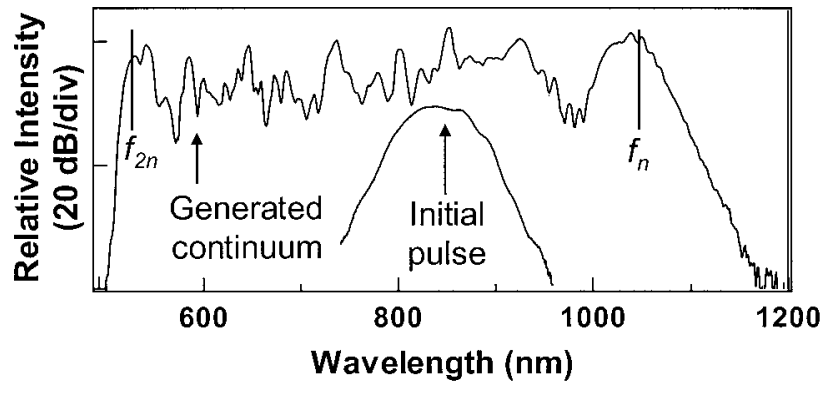

Wavelength $(\mathbf{n m})$

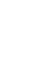




\section{RESEARCH ARTICLES}

from both arms. The entire correlator is in a vacuum chamber held below 300 mTorr to minimize the effect of the dispersion of air. The second-order cross correlation was measured with a two-photon technique (25) by focusing the recombined beam with a spherical mirror onto a windowless GaAsP photodiode. The band gap of GaAsP is large enough and the material purity is high enough so that appreciable single-photon absorption does not occur. This yields a pure quadratic intensity response with a very short effective temporal resolution.

A typical cross correlation is shown in Fig. $5 \mathrm{~A}$. To determine $\Delta \phi$, we fit the fringe peaks of the interferogram to a correlation function assuming a Gaussian pulse envelope. From the fit parameters, we determined the center of the envelope and compared it with the phase of the underlying fringes to find $\Delta \phi$. A fit of the fringe peaks assuming a hyperbolic secant envelope produced nearly identical results. A plot of the experimentally determined relative phases for various offset frequencies, along with a linear fit of the averaged data, is given in Fig. 5B. These results show a small offset of $0.7 \pm 0.35$ rad from the theoretically expected relation $\Delta \phi=4 \pi \delta / f_{\text {rep }}$ (the extra factor of 2 results because the cross correlator compares pulses $i$ and $i+2)$. The experimental slope is within 5\% of the theoretically predicted value and demonstrates our control of the relative carrier-envelope phase. Despite our extensive efforts to match the arms of the correlator, we attribute
Fig. 4. Cross-correlator. The second-order cross correlation between pulse $i$ and pulse $i+2$ is measured to determine the pulse-to-pulse carrier-envelope phase shift. The components inside the shaded box are enclosed 300 mTorr to minimize the dispersive effects of air. The number of mirror bounces in both arms are matched, also to minimize phase errors. The second-order cross correlation is measured with two-photon absorption in a GaAsP photodiode. in a vacuum chamber at

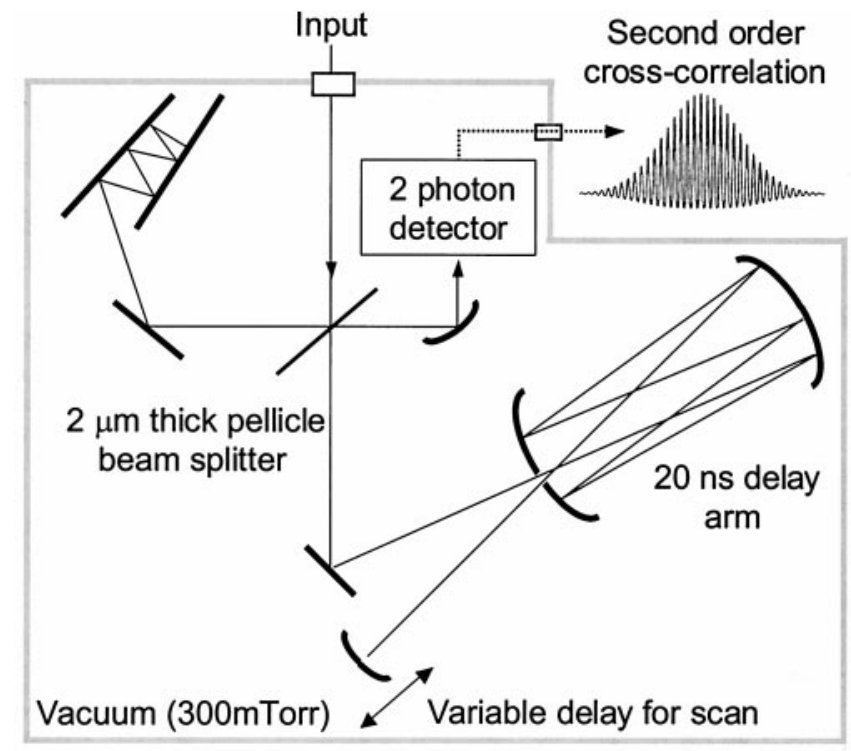

Fig. 5. Correlation results. (A) Typical cross correlation (solid line) between the pulse $i$ and pulse $i+2$, along with a fit of the correlation envelope (dashed line). (B) Plot of the relative phase versus the offset frequency (normalized to the pulse repetition rate). As indicated, a linear fit of our averaged data produces the expected slope of $4 \pi$ with a $5 \%$ uncertainty. The origin of the phase offset is discussed in the text.
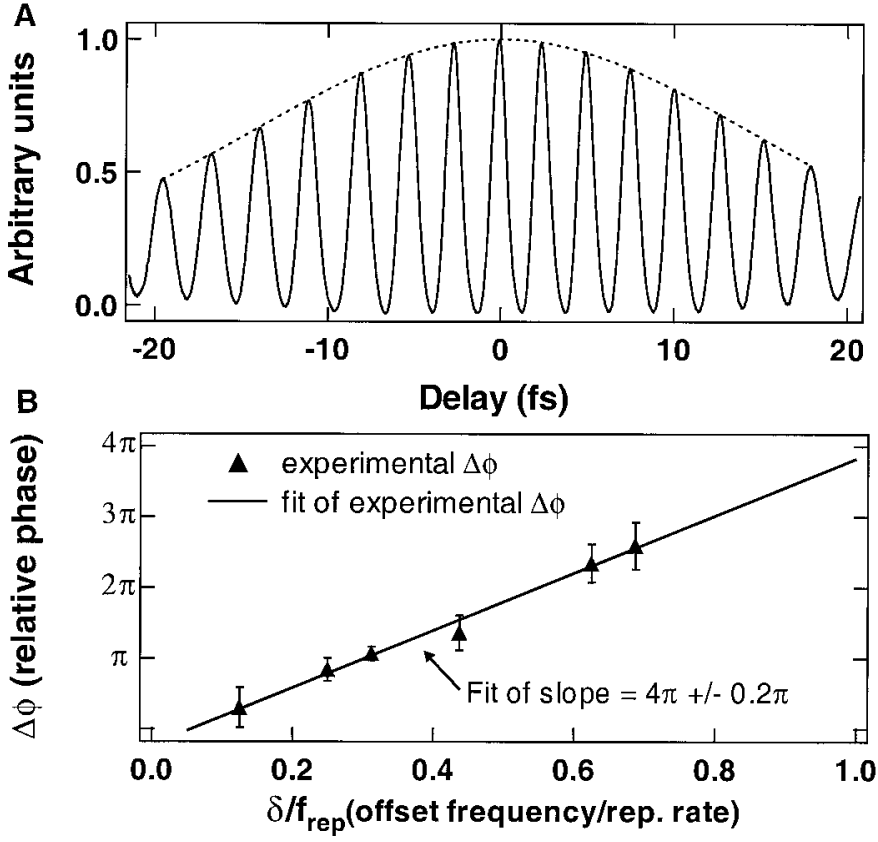

the phase offset between experiment and theory to a phase imbalance in the correlator. The number of mirror bounces in each arm is the same, and mirrors with the same coatings were used for 22 of the 23 bounces in each arm. Nevertheless, because of availability issues, there is a single bounce that is not matched. Furthermore, the large number of bounces necessary to generate the delay means that a very small phase difference per bounce can accumulate and become significant. In addition, the pellicle beam splitter will introduce a small phase error because of the different reflection interface for the two arms. Together, these effects can easily account for the observed offset. The group-phase dispersion due to the residual air only accounts for a phase error of $\sim \pi / 100$. We think that this correlation approach represents the best measurement strategy that can be made short of demonstration of a physical process that is sensitive to the phase.

We think that the uncertainty in the individual phase measurements shown in Fig. 5B arises both from the cross-correlation measurement itself and from environmental perturbations of the laser cavity that are presently beyond the bandwidth of our stabilizing servo loops. Indeed, a measurement in the frequency domain made by counting a locked offset frequency $\delta=19 \mathrm{MHz}$ with 1 -s gate time revealed a standard deviation of $143 \mathrm{~Hz}$, corresponding to a relative phase uncertainty of $10 \mathrm{rad}$. The correlator uses a shorter effective gate time, which decreases the averaging and hence increases the standard deviation. Nevertheless, the uncertainty in the time domain is $10^{3}$ to $10^{4}$ times that in the frequency domain (see below), indicating that the correlator itself contributes to the measurement uncertainty.

With pulses generated by mode-locked lasers now approaching the single-cycle regime $(3,4)$, the control of the carrier-envelope relative phase that we have demonstrated is expected to dramatically impact the field of extreme nonlinear optics. This includes above-threshold ionization and high harmonic generation/x-ray generation with intense femtosecond pulses. Above-threshold ionization with circularly polarized light has recently been proposed as a technique for determining the absolute phase (6). Measurements of x-ray generation efficiency also show effects that are attributed to the evolution of the pulse-to-pulse phase (8).

Absolute optical frequency metrology. In addition to applications in the time domain, the stabilized mode-locked laser shown in Fig. 2 has an immediate and revolutionary impact also in optical frequency metrology. As shown schematically in Fig. 1B, when both the $f_{\text {rep }}$ (comb spacing) and the offset frequency $\delta$ (comb position) are stabilized, lying underneath the broadband continuum envelope is a frequency comb with precisely defined intervals and known absolute frequencies. By stabilizing $f_{\text {rep }}$ 


\section{RESEARCH ARTICLES}

in terms of the primary $9.193-\mathrm{GHz}$ cesium standard, we can then use this frequency comb as a self-referenced "frequency ruler" to measure any optical frequency that falls within the bandwidth of the comb. With this technique, a direct link between the microwave and optical domains is now possible with a single stabilized femtosecond laser.

To demonstrate this application, we present results using this procedure to measure a CW Ti:S laser operating at $778 \mathrm{~nm}$ and locked to the ${ }^{5} \mathrm{~S}_{1 / 2}(\mathrm{~F}=3) \rightarrow{ }^{5} \mathrm{D}_{5 / 2}(\mathrm{~F}=5)$ two-photon transition in ${ }^{85} \mathrm{Rb}$. The experimental setup is shown in Fig. 6A. A portion of the stabilized frequency comb is combined with the 778-nm stabilized Ti:S laser and spectrally resolved with a 1200 lines per millimeter grating. The heterodyne beat between the frequency comb and the $\mathrm{CW}$ stabilized Ti:S laser is measured with a photodiode positioned behind a slit that passes $\sim 1 \mathrm{~nm}$ of bandwidth about $778 \mathrm{~nm}$. By counting both the offset frequency $\delta$ and the heterodyne beat signal between the $\mathrm{CW}$ Ti:S and the comb $\left(f_{\text {beat }}\right)$, the unknown frequency is determined by $f_{\text {unknown }}= \pm \delta+n f_{\text {rep }} \pm f_{\text {beat }}$. The sign ambiguity of $f_{\text {beat }}$ arises because it is not known a priori whether the individual frequency comb member closest to the 778$\mathrm{nm}$ laser is at a higher or lower frequency. A similar ambiguity exists for $\delta$. As the $778-\mathrm{nm}$ frequency is already known within much better than $f_{\text {rep }} / 2=45 \mathrm{MHz}, f_{\text {unknown }}$ is found by simply incrementing or decrementing $n$ and using the appropriate sign of $f_{\text {beat }}$ and $\delta$. Figure 6B displays one set of measurement results relative to the Comite International des Poids et Mesures (CIPM) (1997) recommended value of $385,285,142,378 \pm 5.0$ $\mathrm{kHz}$ for the optical rubidium transition (26). Our first demonstration of this technique is within the uncertainty of the CIPM (1997) val- ue. The width of the Gaussian distribution leads us to suspect that the measurement scatter is most likely due to phase noise in the RF rubidium atomic clock used to stabilize the repetition rate of the laser. Only minimal environmental stabilization of the laser cavity was performed. With a higher quality reference clock, improved environmental isolation of the mode-locked laser cavity and higher bandwidth servo loops, we expect lower amounts of scatter, not only for data such as those presented in Fig. $6 \mathrm{~B}$, but also for time domain results analogous to those in Fig. 5B.

The average of our frequency measurements over several days, giving $-4.2 \pm 1.3$ $\mathrm{kHz}$ from the CIPM (1997) value, agrees quite well with a previous measurement of the JILA rubidium two-photon stabilized reference laser, in which we measured an offset of $-3.2 \pm 3.0 \mathrm{kHz}$ (13). In this previous work, the position of the broadened femtosecond comb was not locked but rather the comb position was calibrated with the fundamental and second harmonic of a secondary, stabilized CW laser, which itself was measured with the octave-spanning comb.

These results demonstrate absolute optical frequency measurements with a single modelocked laser. This technique represents an enormous simplification over conventional frequency metrology techniques, including multiplier chains, and even other femtosecond methods, described earlier. The tools described here should make absolute optical frequency synthesis and measurement a common laboratory practice instead of the heroic effort it has been heretofore.

Conclusion. We have demonstrated stabilization of the carrier phase with respect to the pulse envelope of ultrashort pulses produced by a mode-locked laser using a self-referencing technique that does not require any external
Fig. 6. Frequency metrology with the selfreferenced frequency comb. (A) Experimental setup to measure the ${ }^{5} \mathrm{~S}_{1 / 2}(\mathrm{~F}=3) \rightarrow{ }^{5} \mathrm{D}_{5 / 2}$ $(\mathrm{F}=5)$ two-photon transition in ${ }^{85} \mathrm{Rb}$ with a self-stabilized frequency comb. (B) Histogram of one set of measurements in relation to the recommended CIPM (1997) value of $385,285,142,378 \pm 5.0$ $\mathrm{kHz}$ for the rubidium transition. The standard deviation at the 1-s gate time is $5 \mathrm{kHz}$, which is an absolute uncertainty of 1 part in $10^{11}$.

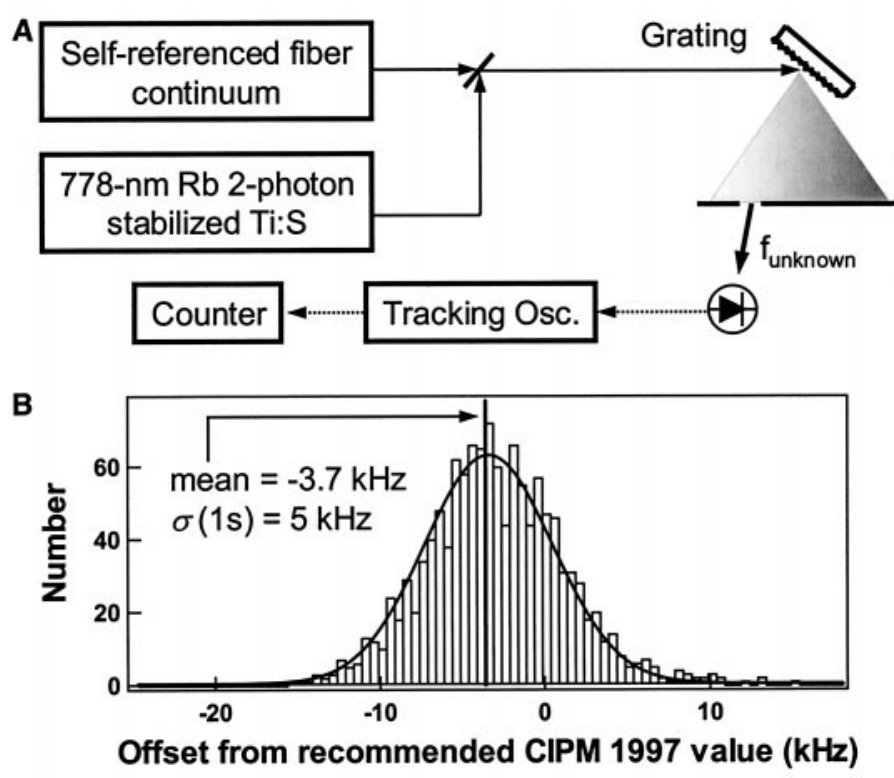

optical input. The phase can either be locked so that every pulse has the identical phase or made to vary so that every $i$ th pulse has the same phase. In the frequency domain, this means that the broad spectral comb of optical lines has known frequencies, namely a simple (large) multiple of the pulse-repetition frequency plus a user-defined offset. This is particularly convenient if the repetition rate of the laser is locked to an accurate microwave or RF clock because then the absolute optical frequencies of the entire comb of lines are known. These results will impact extreme nonlinear optics $(8,27)$, which is expected to display exquisite sensitivity to electric field of the pulse. The self-referencing method also represents a dramatic advance in optical frequency metrology, making measurement of absolute optical frequencies possible with a single laser.

References and Notes

1. G. Steinmeyer, D. H. Sutter, L. Gallmann, N. Matuschek, U. Keller, Science 286, 1507 (1999).

2. M. T. Asaki et al., Opt. Lett. 18, 977 (1993).

3. U. Morgner et al., Opt. Lett. 24, 411 (1999).

4. D. H. Sutter et al., Opt. Lett. 24, 631 (1999).

5. L. Xu et al., Opt. Lett. 21, 2008 (1996).

6. P. Dietrich, F. Krausz, P. B. Corkum, Opt. Lett. 25, 16 (2000).

7. R. J. Jones, J.-C. Diels, J. Jasapara, W. Rudolph, Opt. Commun. 175, 409 (2000)

8. C. G. Durfee et al., Phys. Rev. Lett. 83, 2187 (1999).

9. T. Udem, J. Reichert, R. Holzwarth, T. W. Hänsch, Phys. Rev. Lett. 82, 3568 (1999).

10. , Opt. Lett. 24, 881 (1999).

11. J. Reichert, R. Holzwarth, Th. Udem, T. W. Hänsch, Opt. Commun. 172, 59 (1999).

12. S. A. Diddams, D. J. Jones, L.-S. Ma, S. T. Cundiff, J. L. Hall, Opt. Lett. 25, 186 (2000).

13. S. A. Diddams et al., Phys. Rev. Lett., in press.

14. Various schemes for using mode-locked lasers in optical frequency metrology were recently discussed in work by H. R. Telle et al. [Appl. Phys. B 69, 327 (1999)].

15. See, for example, A. E. Siegman, Lasers (University Science Books, Mill Valley, CA, 1986).

16. J. Ranka, R. Windeler, A. Stentz, Opt. Lett. 25, 25 (2000).

17. J. D. Prestage, R. L. Tjoelker, L. Maleki, Phys. Rev. Lett. 74, 3511 (1995).

18. A. Huber et al., Phys. Rev. Lett. 80, 468 (1998).

19. See, for example, H. Schnatz, B. Lipphardt, J. Helmcke, F. Riehle, G. Zinner, Phys. Rev. Lett. 76, 18 (1996).

20. H. R. Telle, D. Meschede, T. W. Hänsch, Opt. Lett. 15, 532 (1990).

21. P. A. Junger et al., IEEE Trans. Instrum. Meas. 44, 151 (1995).

22. M. Kourogi, K. Nakagawa, M. Ohtsu, IEEE J. Quantum Electron. 29, 2692 (1993).

23. J. L. Hall et al., IEEE Trans. Instrum. Meas. 48, 583 (1999).

24. R. L. Fork, O. E. Martinez, J. P. Gordon, Opt. Lett. 5 , 150 (1984).

25. J. K. Ranka, A. L. Gaeta, A. Baltuska, M. S. Pschenichnikov, D. A. Wiersma, Opt. Lett. 22, 1344 (1997).

26. T. Quinn, Metrologia 36, 211 (1999).

27. Ch. Spielmann et al., Science 278, 661 (1997).

28. The authors gratefully acknowledge J. Ye for help with developing and running the rubidium stabilized Ti:S laser, J. Levine for assistance in establishing common-view global positioning system-based time comparison, and T. W. Hänsch for discussions. Research support from the NIST competence program and the NSF are acknowledged. D.J.J. and S.A.D. are supported by the U.S. National Academy of Sciences/National Research Council postdoctoral fellows program.

13 March 2000; accepted 10 April 2000 
David J. Jones et al.

Science 288, 635 (2000);

DOI: $10.1126 /$ science.288.5466.635

This copy is for your personal, non-commercial use only.

If you wish to distribute this article to others, you can order high-quality copies for your colleagues, clients, or customers by clicking here.

Permission to republish or repurpose articles or portions of articles can be obtained by following the guidelines here.

The following resources related to this article are available online at www.sciencemag.org (this information is current as of July 15, 2015 ):

Updated information and services, including high-resolution figures, can be found in the online version of this article at:

http://www.sciencemag.org/content/288/5466/635.full.html

This article cites 23 articles, 1 of which can be accessed free:

http://www.sciencemag.org/content/288/5466/635.full.html\#ref-list-1

This article has been cited by 752 article(s) on the ISI Web of Science

This article has been cited by 17 articles hosted by HighWire Press; see:

http://www.sciencemag.org/content/288/5466/635.full.html\#related-urls

This article appears in the following subject collections:

Physics, Applied

http://www.sciencemag.org/cgi/collection/app_physics 